\title{
Attosecond Streaking Time Delays: Finite-Range Interpretation and Applications
}

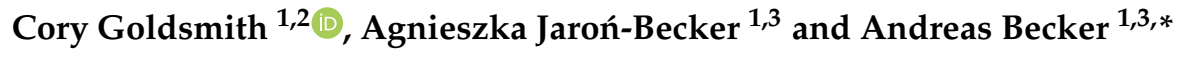 \\ 1 JILA, University of Colorado, Boulder, CO 80309, USA; Cory.Goldsmith@colorado.edu (C.G.); \\ jaron@jila.colorado.edu (A.J.-B.) \\ 2 Department of Chemistry and Biochemistry, University of Colorado, Boulder, CO 80309, USA \\ 3 Department of Physics, University of Colorado, Boulder, CO 80309, USA \\ * Correspondence: andreas.becker@colorado.edu
}

Academic Editors: Mauro Nisoli and Matteo Lucchini

Received: 22 December 2018; Accepted: 22 January 2019; Published: 31 January 2019

\begin{abstract}
We review theoretical studies of the attosecond streaking time delay concept in photoionization via the investigation of the electron dynamics in the streaking field after the transition of the photoelectron into the continuum upon absorption of an extreme ultraviolet photon. Based on the results, a so-called finite range interpretation was introduced, which highlighted that the delay is accumulated until the streaking pulse ends and, hence, over a finite range of the potential of the parent ion. Following a discussion of the analysis leading to this interpretation, we summarize a few applications which provide insights into different aspects of the streaking time delay concept in photoionization. Besides a review of previously presented results, we give an analysis of the relevance of the first half-cycle of the streaking field and an outlook regarding the perspective of using the streaking method to resolve dynamical changes in the potential that the photoelectron explores during its propagation in the continuum.
\end{abstract}

Keywords: ultrafast laser science; photoionization; attosecond measurement techniques; streaking time delays

\section{Introduction}

To resolve dynamics in matter on ultrashort time scales, a variety of pulse technologies, e.g., ultrashort electron pulses, femtosecond $\left(1 \mathrm{fs}=10^{-15} \mathrm{~s}\right)$ and attosecond ( 1 as $\left.=10^{-18} \mathrm{~s}\right)$ laser pulses, and X-ray free electron laser pulses have been developed. Among these, attosecond pulses are, in general, not yet intense enough to be used in pump-probe measurements. To gain resolution on the attosecond time scale, a number of methods have been explored in which a combination of an attosecond extreme ultraviolet (XUV) pulse and a second femtosecond (near-)infrared (IR) laser pulse is applied (for recent overviews, see e.g., [1-3]). One of these techniques is the attosecond streak camera method [4], in which an attosecond pulse photoionizes a target (via the absorption of a single photon) and a few-cycle infrared pulse is used to modulate the final momentum of the photoelectron. By varying the relative delay between the two pulses, an oscillating streaking pattern for the final photoelectron momentum $\mathbf{k}_{f}$ is observed, which can be represented by:

$$
\mathbf{k}_{f}^{(n, l)}\left(t_{i}\right)=\mathbf{k}_{0}-\mathbf{A}_{s}\left(t_{i}+\Delta t_{s}^{(n, l)}\right),
$$

where $\mathbf{k}_{0}$ is the field-free momentum of the electron, initially bound in the $|n, l\rangle$ state of an atom, upon release into the streaking field, represented by the vector potential $\mathbf{A}(t)$, at time $t_{i}$. The offset $\Delta t_{s}^{(n, l)}$ with respect to the release time of the photoelectron in the argument of the vector potential is typically 
referred to as the streaking time delay. Hence, the time delay is visualized as the offset in the streaking trace of the momentum of the photoelectron propagating in the Coulomb potential as compared to that of a free photoelectron (c.f., Figure 1). We note that, in an attosecond streaking experiment (e.g., [5]), the temporal information is usually gained via the comparison of streaking traces from photoelectrons exposed to the same streaking field, but which populated different orbitals initially. This corresponds to the difference of streaking time delays from the two states involved (c.f., Figure 1). However, in theoretical analysis, the focus of studies is primarily the streaking time delay $\Delta t_{s}^{(n, l)}$, as defined in Equation (1). Since in the remainder of the article the initial state is typically implied, we simply refer to this quantity as $\Delta t_{s}$ below.

Various theoretical work has established that the streaking time delay obtained in numerical simulations can be written as a sum:

$$
\Delta t_{S}=\Delta t_{W S}+\Delta t_{C L C}
$$

where $\Delta t_{W S}$ is the Wigner-Smith time delay $[6,7]$ and $\Delta t_{C L C}$ is a term that accounts for the coupling between the streaking field and long-range Coulomb potential. Justification of this approach as well as theoretical analysis of additional effects, such as multielectron contributions or the role of the attochirp, have been discussed in review articles recently (e.g., [8-10]). Here, we focus on a review of recent analysis of the streaking time delay via the investigation of the electron dynamics in the streaking field after transition of the photoelectron into the continuum upon absorption of an XUV photon. Classical estimates and predictions of model formulas related to this analysis have been found to be in good agreement with results of ab initio numerical simulations. This established a basis to use the approach to gain insights into the interpretation and analysis of various aspects of the streaking time delay in photoionization. Specifically, it leads to the interpretation that the delay in photoionization is only accumulated until the streaking pulse ceases. Hence, the streaking measurement probes the (long range) potential of the parent ion over a finite range only. While we focus here on interpretation and applications related to streaking time delays in one-photon ionization, we may note that recently the extension of the streaking concept to two-photon ionization within the present approach [11,12] as well as in other context [13-16] has been considered.
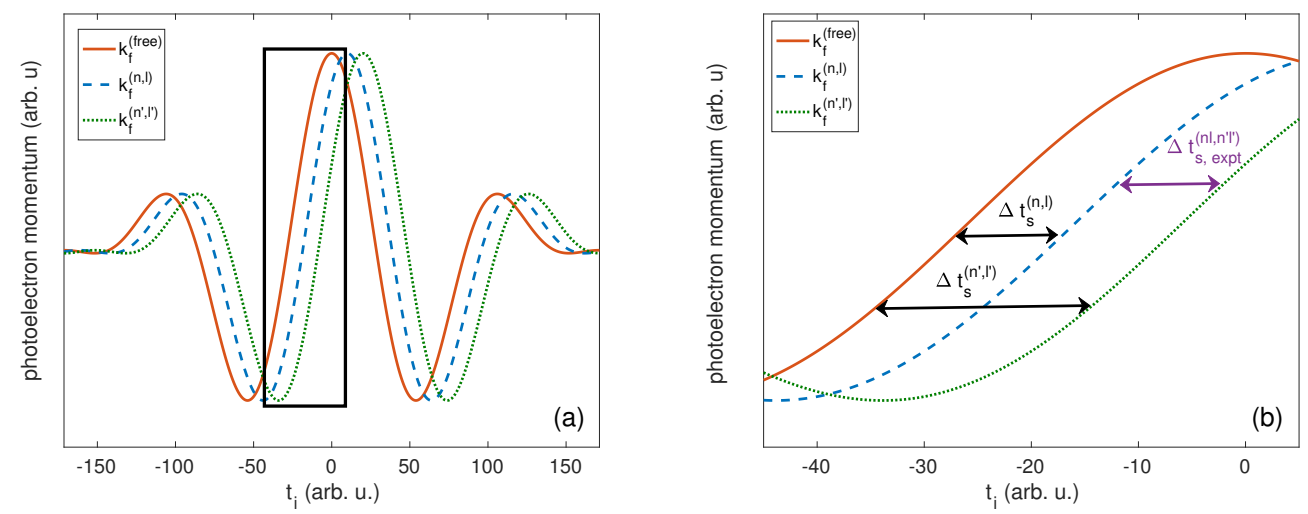

Figure 1. Conceptual visualization of the different streaking time delays in photoionization, namely the experimentally observed delay $\Delta t_{s, \text { expt }}^{\left(n l, n^{\prime} l^{\prime}\right)}$ between streaking traces for emission from initial states $\mid n, l>$ and $\mid n^{\prime}, l^{\prime}>$ and the theoretically considered delays $\Delta t_{S}^{(n l)}$ and $\Delta t_{s}^{\left(n^{\prime} l^{\prime}\right)}$ with respect to the streaking trace of a free electron. Panel (b) is an enlargement of the full traces shown in panel (a).

Before proceeding, we may briefly mention that results of ab initio numerical calculations as well as other theoretical work showed a significant discrepancy with the data of the original streaking experiment [5]. Only recently, new insights into the origin of this disagreement has been achieved via an alternative measurement [17] based on the RABBITT (Reconstruction of attosecond beating 
by interfering two-photon transitions, [18]) technique. Due to the better spectral resolution in the RABBITT experiment signals from the direct photoionization and a shake-up process, in which a second electron is excited along with the photoionization of the first (streaked) electron, could be disentangled, which overlapped in the original streaking experiment. The time delay measurement for the photoionization signal in the RABBITT experiment is in much better agreement with the theoretical predictions.

The article is organized as follows: in Section 2, we briefly discuss the finite range interpretation, which arises from a classical analysis of the streaked electron dynamics and comparison with results of quantum simulations. Furthermore, we show that the finite-range feature allows for developing an analytical formula, which provides accurate estimates of the time delay. Beyond this capability, applications of the formula provide insights in different aspects of the streaking time delay, which we will discuss in Section 3. The latter part includes, besides a review, discussion of the relevance of the first half-cycle of the streaking field (Section 3.2) and the resolution of dynamics (Section 3.4), which have not been analyzed before. We end with a brief summary.

\section{Finite-Range Interpretation}

\subsection{Analysis of Streaking Time Delays Using Classical Electron Dynamics}

It has been shown (e.g., $[19,20]$ ) that predictions for the streaking time delay determined by solving the classical dynamics of the electron ionized by the XUV field and streaked by the IR field are in good agreement with results from ab initio solutions of the time-dependent Schrödinger equation (TDSE) simulating a streaking measurement. For further analysis of the physical meaning of the time delay, one may therefore consider the solution of the Newton equation for the propagation of the photoelectron in the streaking field after its transition in the continuum:

$$
\frac{d \mathbf{k}(\mathbf{t})}{d t}=-\mathbf{E}_{s}(t)-\nabla V(\mathbf{r})
$$

In Equation (3), $\mathbf{k}(t)$ represents the momentum of the photoelectron, $\mathbf{E}_{s}$ is the streaking field, and $V(\mathbf{r})$ is the Coulomb potential of the residual atom (in the single-active-electron approximation). For a linearly polarized streaking field the interaction with the streaking field takes effect along the direction of the laser polarization, say $z$-direction, and the corresponding component of the final momentum $k_{f, z}$ can be written as:

$$
k_{f, z}\left(t_{i}\right)=\sqrt{k_{0, z}^{2}-2 \int_{t_{i}}^{T_{s}} E_{s}(t) k_{z}(t) d t}=k_{0, z}-A_{s}\left(t_{i}+\Delta t_{s}\right),
$$

where $k_{0, z}$ is the field-free asymptotic momentum, $t_{i}$ is the time of transition of the photoelectron in the continuum, $A_{s}$ and $T_{S}$ are the vector potential and duration of the streaking field, and $\Delta t_{S}$ is the streaking time delay, as in Equation (1).

Assuming that the streaking field is weak, the vector potential in Equation (4) can be expanded as:

$$
A_{s}\left(t_{i}+\Delta t_{s}\right) \simeq A_{s}\left(t_{i}\right)-E_{S}\left(t_{i}\right) \Delta t_{s},
$$

and the streaking time delay can be written as (for $E_{s}\left(t_{i}\right) \neq 0$ ):

$$
\Delta t_{s} \simeq \frac{\sqrt{k_{0, z}^{2}-2 \int_{t_{i}}^{T_{s}} E_{\mathcal{S}}(t) k_{z}(t) d t}-k_{0, z}+A_{s}\left(t_{i}\right)}{E_{s}\left(t_{i}\right)} .
$$


This expression can be further simplified by noting that the momentum shift is usually small if the streaking field is weak, and expanding the square root in Equation (6) to first order to obtain (for $\left.E_{s}\left(t_{i}\right) \neq 0\right)[20,21]$ :

$$
\Delta t_{s} \simeq \frac{1}{E_{s}\left(t_{i}\right)} \int_{t_{i}}^{T_{s}} E_{s}(t)\left[1-\frac{k_{z}(t)}{k_{0, z}}\right] d t .
$$

Although Equation (7) already provides the interpretation of the streaking time delay as a finite-range time delay, it has been sometimes found instructive to also write it using a finite-difference approximation (for $E_{s}\left(t_{i}\right) \neq 0$ ):

$$
\Delta t_{s} \simeq \sum_{j=1}^{N} \frac{E_{s}\left(t_{j}\right)}{E_{S}\left(t_{i}\right)}\left[1-\frac{k_{z}(t)}{k_{0, z}}\right] \delta t \simeq \sum_{j=1}^{N} \frac{E_{s}\left(t_{j}\right)}{E_{s}\left(t_{i}\right)} \Delta t_{\mathrm{ff}}^{(j)},
$$

in which one assumes that the streaking field and the electron momentum are approximately constant for the short time intervals $\left[t_{j}, t_{j}+\delta t\right]=\left[t_{j}, t_{j+1}\right]$ while propagating over the short distance $\left[r_{j}, r_{j}+\delta r\right]=$ $\left[r_{j}, r_{j+1}\right]$, i.e., $E_{\mathcal{S}}(t) \simeq E_{\mathcal{S}}\left(t_{j}\right)$ and $k(t) \simeq k\left(t_{j}\right)$. In the last part of Equation (8), we identified the term in the square brackets times $\delta t$ to be approximately equal to the instantaneous field-free time delay $\Delta t_{f f}^{(j)}$, which the electron accumulates in the potential $V$ as compared to the propagation of a free particle.

The field-free time delays can be also written as

$$
\Delta t_{\mathrm{ff}}^{(j)}=\Delta t_{\mathrm{ff}}\left(k_{j+1}, r_{j+1}\right)-\Delta t_{\mathrm{ff}}\left(k_{j}, r_{j}\right)
$$

where $\Delta t_{f f}(k, r)$ is the field-free time delay accumulated during the propagation from the origin of the transition into the continuum to location at a finite distance $r$ with momentum $k=k(r)=$ $\sqrt{2\left(E_{a s y m}-V(r)\right)}$ and $E_{a s y m}$ is the asymptotic kinetic energy of the photoelectron. It has been found [20-23] that the classical estimates for the streaking time delays remain approximately constant while varying $t_{i}$ over one field cycle. However, we point out that, independent of the representation of the streaking time delay as integral (Equation (7)) or in its finite-difference approximation (Equation (8)), these classical predictions diverge for $E_{S}=0$ and, hence, are not applicable at the corresponding times $t_{i}$. For further discussion of the derivation, please see Refs. [20,21].

In order to apply the classical estimates, the corresponding predictions have to be validated against results of ab initio numerical calculations. It has been shown $[11,12,20,21]$ that the classical results, based on Equations (7) and (8), are in good agreement with ab initio TDSE results for the streaking time delay. This is exemplified in Figure 2 for (a) hydrogen atoms, (b) helium atoms and (c) neon atoms, where the classical estimates (solid lines) are compared with the results of ab initio calculations from Refs. [24,25]. Please note that Figure 2 also includes a comparison with predictions from an analytical formula (dashed lines), which will be discussed in Section 2.3. The level of agreement within the range of a few attoseconds provides the basis to use the classical analysis to gain insights into an interpretation and various aspects of the streaking time delay. 

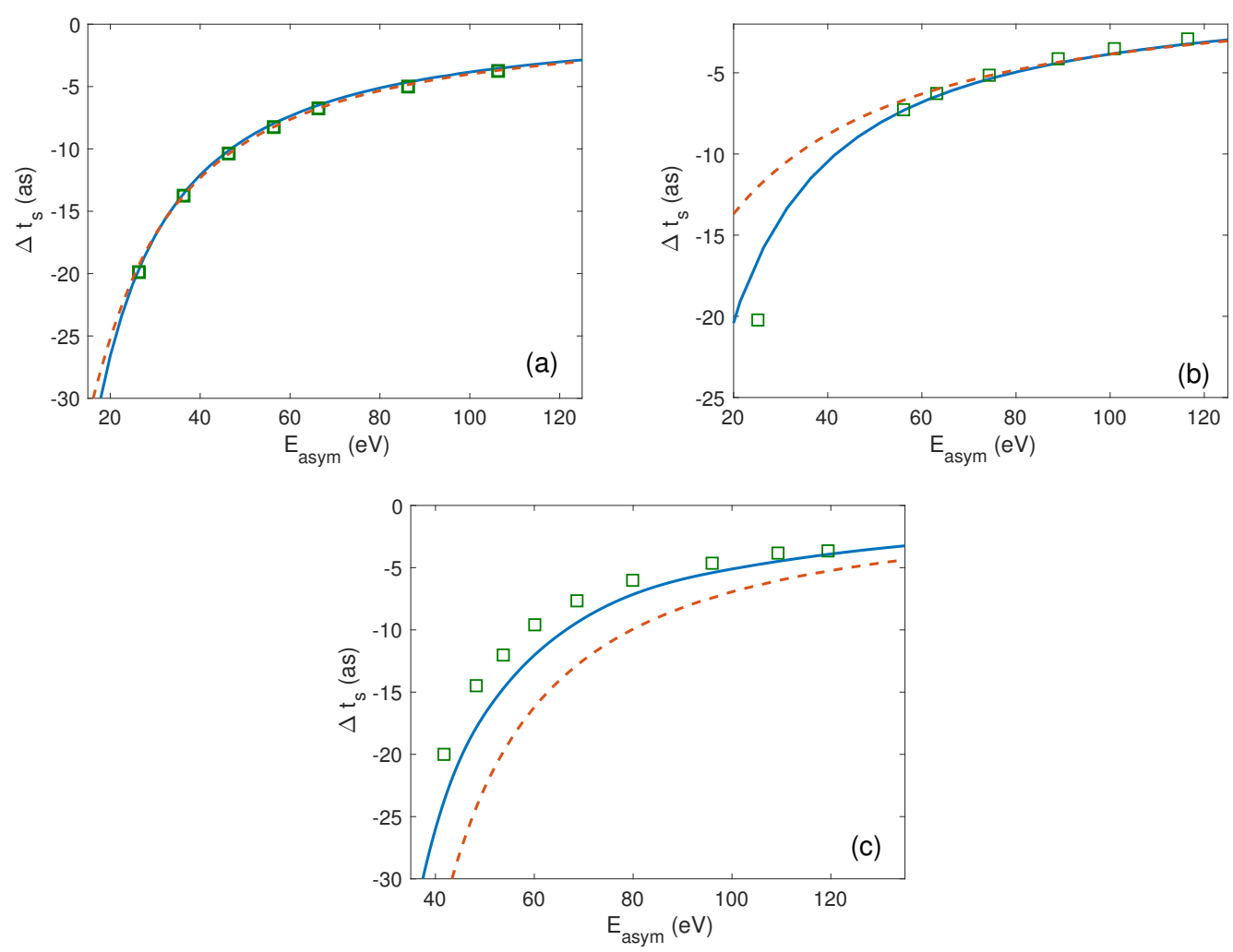

Figure 2. Streaking time delays for hydrogen atom (a); helium atom (b); and neon atom (c): a comparison of results of ab initio calculations is shown (open squares, taken from [24] for hydrogen and helium, [25] for neon), classical calculations (solid lines, Equation (8)) and predictions of analytical model formula (dashed lines, Equation (11)). For neon atoms, only the d-channel is considered in the analytical calculation.

\subsection{Accumulation over Finite Time and Finite Range}

The formulas, Equations (7) and (8), give an interesting interpretation of the streaking time delay, namely that it can be viewed as the field-free time delay $\Delta t_{\mathrm{ff}}$, obtained by a photoelectron without the streaking field, weighted by the (instantaneous) streaking field strength as the photoelectron propagates through the potential. An important consequence of this interpretation is that the time delay is determined by the electron over a finite time interval between the time of transition in the continuum $t=t_{i}$ and the end of the streaking pulse at $t=T_{s}$. During this time interval, the photoelectron explores a finite range in space only.

This interpretation has been tested in quantum streaking simulations for photoemission of an electron initially bound in a 1D model potential [20]. To illustrate the conclusions from that work, we consider here calculations using the model potential:

$$
V_{x}=-\frac{Z}{\sqrt{x^{2}+\alpha}}+V_{0} \exp ^{\left.-\left(|x|-x_{0}\right) / \sigma\right)^{2}},
$$

which consists of a (soft-core) Coulomb potential with $\alpha=2.0$ centered at the origin and an effective charge $Z=3$ and an additional Gaussian-shaped potential with depth $V_{0}=-0.5$ a.u. and width $\sigma=2.0$ centered at a distance $x_{0}=850$ a.u. from the center of the Coulomb potential. Choosing the initial state to be the ground state of the Coulomb potential solutions of the corresponding 1D TDSE in the fields of a XUV pulse and an IR streaking pulse have been obtained and the streaking time delay has been extracted from the results (for methods to obtain streaking time delays from simulations, see e.g., [21]). Each of the calculations has been performed over a long propagation times such that the 
outgoing photoelectron wavepacket reaches distances of more than 1000 a.u., i.e., distances beyond the range of the Gaussian potential, at the end of the simulation.

In Figure 3b, we compare the results for the streaking time delay with (stars) and without (circles) the additional Gaussian potential as a function of the number of cycles of the streaking pulse. For short streaking pulses, the delay is found to be essentially identical with and without the Gaussian potential. In each of these simulations with short pulses, the center of the outgoing wavepacket is located at the $x<x_{0}=850$ a.u. at the end of streaking pulse (Figure 3a). In contrast, as soon as the center of the photoelectron wavepacket reaches the Gaussian potential, centered at $x=850$ a.u., before the end of the pulse, the results from the simulations with and without Gaussian potential differ. Thus, the streaking time delay accounts for the presence of the Gaussian potential only when the electron wave packet reaches the potential before the interaction with the streaking pulse ceases, in agreement with the finite-range interpretation put forward based on the classical analysis.
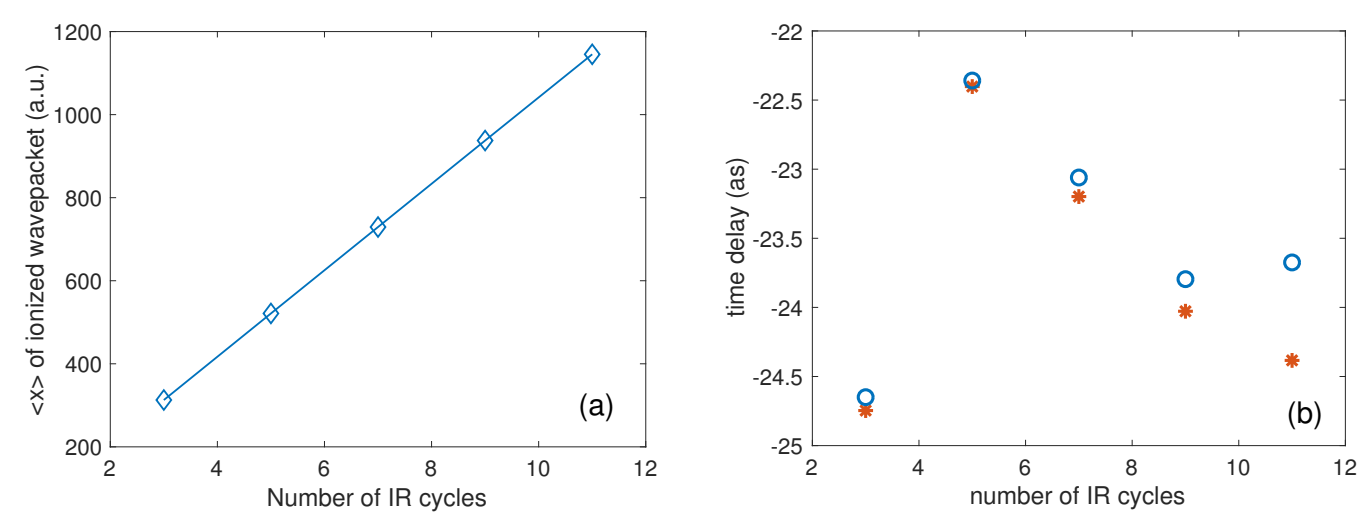

Figure 3. (a) Range of wavepacket propagation in an $800 \mathrm{~nm}$ streaking pulse after emission at the peak of the pulse as a function of the number of cycles for 1D soft-core model; (b) streaking time delay in a potential with additional perturbation (stars) at $x_{0}=850$ a.u. as compared to the delay obtained without the additional potential (circles) as a function of the number of cycles.

\subsection{Analytical Formula}

Based on the interpretation that the streaking time delay is accumulated by the photoelectron over a finite time only, and hence can be understood as a probe of the (long-range) atomic potential over a finite distance, one can proceed to consider cut-off potentials for a further analysis of the time delay. In order to do so, one may assume a classical-quantum correspondence for the field-free time delay, in which the classical estimate, Equation (8), is decomposed in a sum as [23]:

$$
\Delta t_{s} \simeq \sum_{j=1}^{N} \frac{E_{s}\left(t_{j}\right)}{E_{s}\left(t_{i}\right)}\left[\Delta t_{\text {short }}^{(j)}+\Delta t_{\text {phase }}^{(j)}+\Delta t_{\text {log }}^{(j)}\right],
$$

where the terms in the brackets are the well-known short-range, Coulomb-phase and logarithmic contributions to the quantum field-free time delay. Note that each of the terms represents the derivative of a contribution to the quantum mechanical phase shift with respect to the photoelectron's energy.

For example,

$$
\Delta t_{\text {phase }}(k, r)=\frac{1}{k} \frac{\partial}{\partial k} \arg [\Gamma(1+l+i \eta)]
$$

and

$$
\Delta t_{\log }(k, r)=\frac{1}{k} \frac{\partial}{\partial k}\left[\frac{Z}{k} \ln (2 k r)\right]
$$

where $l$ is the angular momentum of the photoelectron, $\eta=-Z / k$, and $Z$ is the charge of the residual ion. Specifically, $\Delta t_{\text {short }}$ is the contribution related to the short-range part of the atomic potential, 
while the other two terms result from the scattering off the $-Z / r$ part of the ionic potential for nuclear charge $Z$. Note that, for an infinite range, Coulomb potential $\Delta t_{\text {log }}$ diverges, while $\Delta t_{\text {phase }}$ converges. Therefore, one typically refers to the sum of the short-range and Coulomb-phase term as the widely known Wigner-Smith time delay $\Delta t_{W S}$ in the context of scattering off an ionic core with a long-range Coulomb potential $[6,7]$.

Each of the three contributions in Equaiton (11) can then be again written as a difference

$$
\Delta t_{i}^{(j)}=\Delta t_{i}\left(k_{j+1}, r_{j+1}\right)-\Delta t_{i}\left(k_{j}, r_{j}\right),
$$

where $\Delta t_{i}(k, r)$ ( $i=$ short, phase, $\left.\log \right)$ is the respective contribution to the field-free time delay accumulated during the propagation from the origin of the transition into the continuum to location at a finite distance $r$ with momentum $k=k(r)=\sqrt{2\left(E_{a s y m}-V(r)\right)}$. Thus, the terms $\Delta t_{i}(k, r)$ correspond to time delay contributions in a potential which is cut-off at $r$. Consequently, due to the finite-range characteristic, none of the terms, including the logarithmic term, diverge here. This has the advantage that the corresponding phase-shift in the wavefunction is finite and one does not encounter conceptual issues like the divergence of the logarithmic term in a Coulomb potential.

It has been shown [23] that each of the terms in Equation (11) can be evaluated analytically for an atom using standard methods, such as the distorted Born approximation and the single-active-electron approximation. Estimates based on the analytical formula (dashed lines, Equation (11)) are found to be in good agreement with the results of ab initio simulations and those of classical calculations for the streaking time delay (see Figure 2, [23]). This validation puts us in the position to use this analytical formula for further analysis of certain aspects of the streaking time delay.

\section{Applications}

The finite-range interpretation and, specifically, the validation of the classical and analytical formulas, Equations (8) and (11), against results of ab initio quantum simulations provide the opportunity to obtain estimates for the streaking time delay with good accuracy in short computation time. In this section, we review applications of this approach that enable additional insights into electron dynamics gained by determination of the streaking time delay.

\subsection{Relation to Wigner-Smith Time Delay}

As mentioned in the Introduction, the streaking time delay is often represented as a sum of the Wigner-Smith time delay and a Coulomb-laser coupling term. To gain further insights into this interpretation, the analytical formula, Equation (11), has been used to study the effect of the streaking field on each of the three contributions (short-range, Coulomb phase and logarithmic) [23]. The absolute difference of the results obtained for each contribution with and without considering the prefactor $E_{s}\left(t_{j}\right) / E_{s}\left(t_{i}\right)$ in Equation (11) is shown as a function of XUV photon energy in Figure 4. The results reveal that for the short-range (a) and the Coulomb phase (b) terms, the effect of the streaking field is significant at photon energies near the threshold only. On the other hand, the effect on the logarithmic term (c) is much larger.

Based on this comparison, the analytical estimate of the time delay, Equation (11), can be further approximated as:

$$
\Delta t_{s} \simeq \Delta t_{\text {short }}+\Delta t_{\text {phase }}+\sum_{j=1}^{N} \frac{E_{S}\left(t_{j}\right)}{E_{S}\left(t_{i}\right)} \Delta t_{\text {log }}^{(j)} \simeq \Delta t_{W S}+\Delta t_{C L C}
$$

where $\Delta t_{W S}=\Delta t_{\text {short }}+\Delta t_{\text {phase }}$ represents the Wigner-Smith time delay, while $\Delta t_{C L C}=\sum_{j=1}^{N} \frac{E_{s}\left(t_{j}\right)}{E_{s}\left(t_{i}\right)} \Delta t_{\log }^{(j)}$ can be interpreted as the Coulomb-laser coupling term. Thus, the finite-range interpretation provides 
an alternative route to justify the widely used decomposition of the streaking time delay into the Wigner-Smith term and Coulomb-laser coupling term.
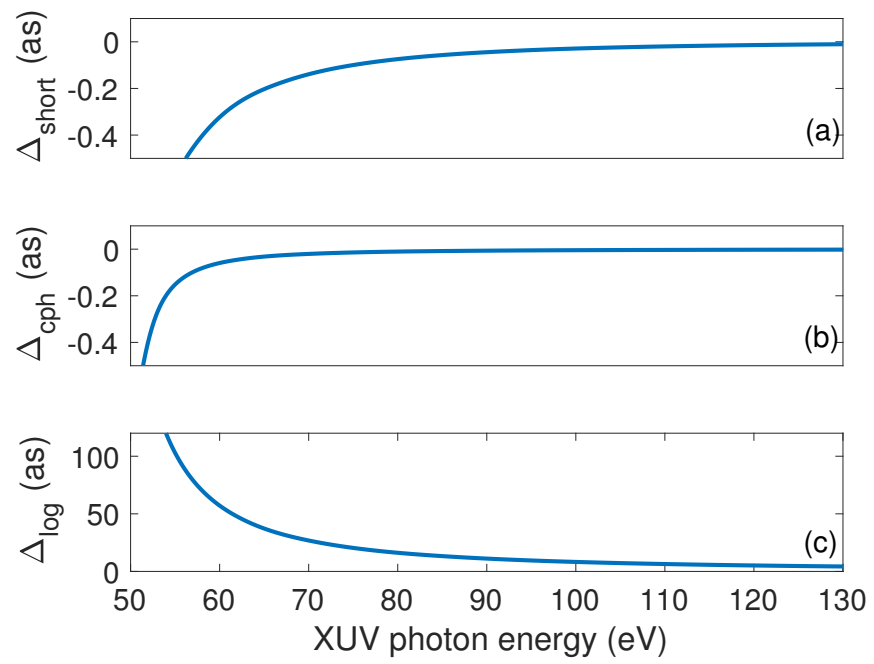

Figure 4. Absolute difference for the different contributions to the time delay of neon atom with and without the effect of the streaking field as a function of electron energy and streaking field wavelength: (a) short-range contribution; (b) Coulomb phase contribution; and (c) Coulomb logarithmic contribution. Parameters of the streaking field: wavelength $\lambda_{s}=800 \mathrm{~nm}$, peak intensity $I_{S}=1 \times 10^{12} \mathrm{~W} / \mathrm{cm}^{2}$, and pulse duration of three cycles is used [23].

\subsection{Relevance of the First Half-Cycle of Streaking Field}

In an analysis of a RABBITT experiment, it has been recently suggested [26] that the information extracted from the measurement mainly depends on the dynamical interactions during the first half cycle of the IR field after the transition of the electron into continuum at $t_{i}$. Since it has been shown [8] that RABBITT and streaking measurements provide equivalent information concerning the time delay, the model implies that the observed streaking (or RABBITT) time delay is essentially determined within one half cycle of the streaking field, i.e., according to the finite-range model between $t_{i}$ and $t_{i}+T / 2$, where $T$ is the period of the streaking field. Both the classical estimates, Equations (7) and (8), or the analytical formula, Equation (11), allow for testing this assumption used in the model by Kiesewetter et al. For example, the streaking time delay can be evaluated as a function of time after transition in the continuum from the expression in Equation (7) by varying the upper limit of the integral between $t_{i}$ and $T_{s}$, i.e.,

$$
\Delta t_{S}\left(t^{\prime}-t_{i}\right)=\frac{1}{E_{S}\left(t_{i}\right)} \int_{t_{i}}^{t^{\prime}} E_{S}(t)\left[1-\frac{k_{z}(t)}{k_{0, z}}\right] d t .
$$

Equivalently, in the finite-difference approximations, Equations (8) and (11), the upper limit of the sum can be varied to obtain $\Delta t_{s}\left(t^{\prime}-t_{i}\right)$. In the present study, we have considered streaking of the photoionization of the hydrogen atom, applied the analytical formula, Equation (11), and have chosen the time of ionization $t_{i}$ to coincide with the peak of the streaking pulse.

In Figure $5 \mathrm{a}$, we present the accumulated streaking time delay $\Delta t_{s}$ as a function of time, $t^{\prime}-t_{i}$, after the transition in the continuum for a streaking wavelength of $800 \mathrm{~nm}$ and seven different XUV central frequencies. To guide the eyes and, for further comparison, the first quarter cycle, $t^{\prime}-t_{i}=T / 4=27.5$ a.u. (vertical blue dashed line), and the first half cycle, $t^{\prime}-t_{i}=T / 2=55$ a.u., are also marked (vertical black dashed line), after liberation of the photoelectron. 
We first discuss the general trend of the results in Figure 5a. It is seen that in each case the streaking time delay oscillates as a function of time before it converges towards its final value. This can be understood as follows: in the region $0 \leq t^{\prime}-t_{i} \leq T / 4$, the influence of the Coulomb potential is strong, and, accordingly, there is a sharp increase in the magnitude of the time delay. Beyond $t^{\prime}-t_{i}=T / 4$, the field changes sign and then oscillates until the end of the pulse. Consequently, the accumulated streaking time delay as a function of time oscillates as well. The magnitude of the oscillation decreases since the amplitude of the streaking electric field decreases (reducing the weight of the field-free time delays in Equation (11)) and the Coulomb potential decreases in strength (reducing the magnitude of the field-free time delays in Equation (11)).

In order to quantify how fast the time delay is accumulated towards its final value, we have further determined times beyond which the magnitude of the streaking time delay remains within a certain range of its final value. For example, $t_{90 \%}$ is defined as

$$
\left|\Delta t_{s}\left(t^{\prime}-t_{i}\right)\right| \leq 0.9\left|\Delta t_{s}\left(T_{s}-t_{i}\right)\right| \quad \text { for } \quad t^{\prime} \geq t_{90 \%}
$$

and $t_{99 \%}$ is analogous. The times $t_{90 \%}$ (stars) and $t_{99 \%}$ (circles) are marked on each of the curves in Figure 5a. As one can see from the comparison, in each case $t_{90 \%}<T / 2$, which means that the accumulated time delays $\Delta t_{s}$ reaches $90 \%$ of the final value before the end of the first half cycle of the streaking field after transition of the photoelectron in the continuum. Furthermore, it is seen that the rate of convergence within the first half cycle of the streaking field improves as the XUV photon energy increases. The reason for this trend is that, as the kinetic energy of the photoelectron becomes higher, the faster it propagates away from the core. Therefore, by the time the sign of the field changes, due to the large decrease in the magnitude of the Coulomb potential, the remaining terms in the sum are sufficiently small such that they do not contribute significantly to the total value. We have found a similar trend (not shown) if the wavelength of the streaking field is increased.

The conclusion that the accumulated streaking time delay achieves $90 \%$ of its final value within the first half cycle of the streaking pulse holds independent of the streaking pulse duration. This can be seen from the results in Figure $5 b$, in which the times $t_{90 \%}$ are presented for different pulse durations (symbols) as a function of the XUV frequency. For comparison, $T / 4=27.5$ a.u. (horizontal green lines) and $T / 2=55$ a.u. (horizontal blue dashed line) are also indicated. Again, for each case studied, we find that $t_{90 \%}<T / 2$. Therefore, overall the results of our theoretical analysis support the assumption by Kiesewetter et al. [26] that the time delay is essentially obtained within the first half-cycle of the streaking field after the transition of the photoelectron in the continuum.

Finally, we also consider the different contributions to the time delay (short-range, Coulomb-phase and logarithmic, see Equation (11)), separately. In Figure 5c, we compare the times at which each of these contributions reaches $90 \%$ of its final value with each other and with the time $t_{90 \%}$ for the total accumulated time delay. The results clearly show that it is the long-range logarithmic term (diamonds) that essentially determines the time after transition of the photoelectron in the continuum, at which the full time delay (stars) approaches its final value. On the other hand, the short-range (circles) and Coulomb (squares) terms converge much faster, clearly within much less than one quarter cycle of the streaking pulse (solid line). This is in agreement with the results discussed in Section 3.1 that the streaking pulse has a small effect on these two contributions to the streaking time delay. Furthermore, it supports another assumption in Ref. [26] that the information about the differences between short-range parts of different atomic potentials is incorporated in the streaking time delay accumulated within the first quarter cycle of the streaking field after emission of the photoelectron in the continuum. 

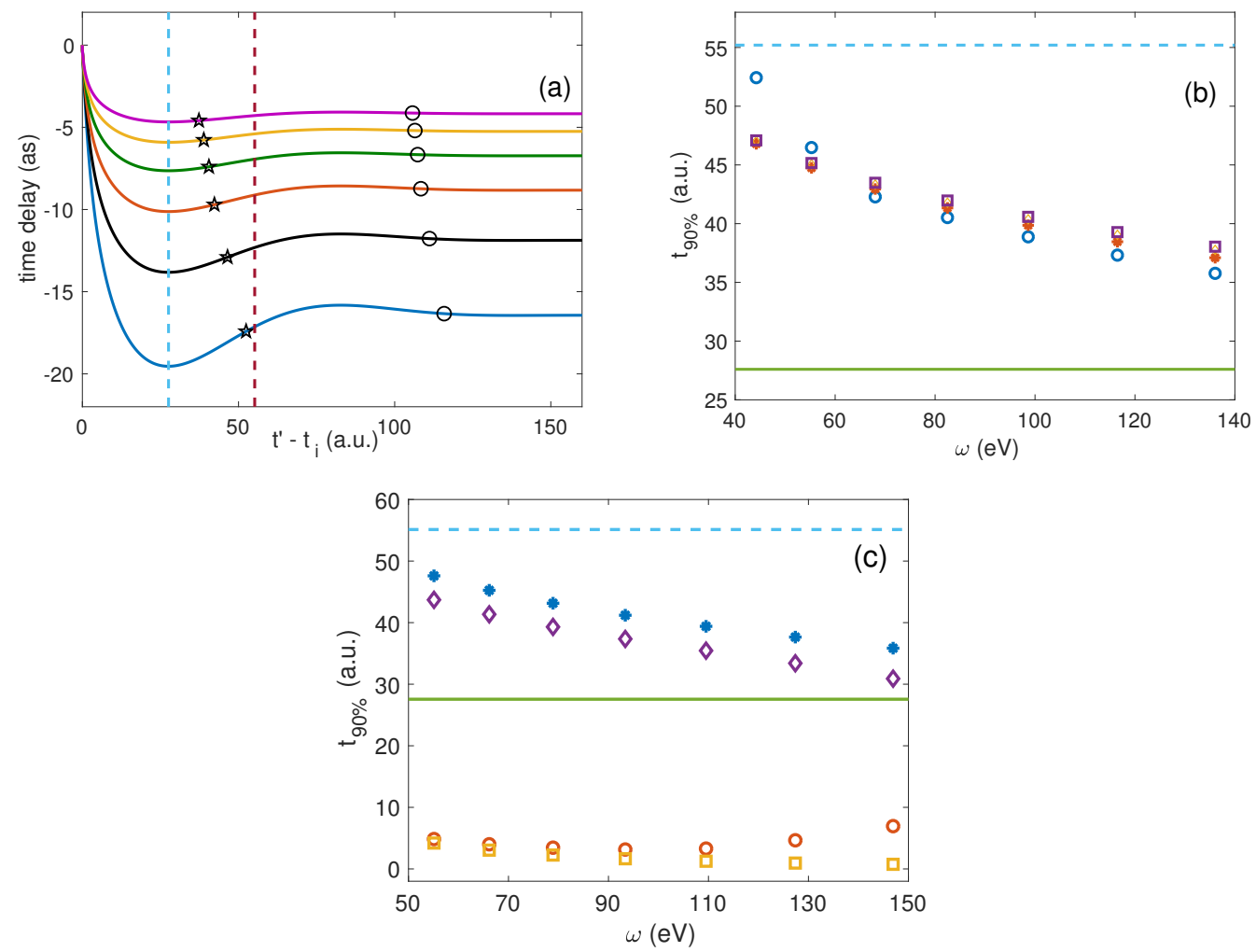

Figure 5. (a) Analytical predictions for the streaking time delay (in attoseconds) of hydrogen atom as a function of time $t^{\prime}-t_{i}$ (in atomic units) for electron kinetic energies corresponding to XUV central frequencies of $\omega=44.22 \mathrm{eV}$ (blue), $\omega=55.27 \mathrm{eV}$ (black), $\omega=68.03 \mathrm{eV}$ (red), $\omega=82.48 \mathrm{eV}$ (green), $\omega=98.64 \mathrm{eV}$ (yellow), and $\omega=116.49 \mathrm{eV}$ (pink) and a three-cycle streaking pulse. The times $t_{90} \%$ and $t_{99 \%}$ are shown as stars and circles, respectively, and $T / 4$ and $T / 2$ are denoted with dashed vertical lines; (b) time $t_{90 \%}$ (in atomic units) is shown as a function of XUV central frequency for different streaking pulse durations; $N_{s}=3$ cycles (circles), $N_{s}=4$ cycles (stars), $N_{s}=5$ cycles (diamonds), and $N_{s}=6$ cycles (squares). Further shown is $T / 2$ (dashed light blue) and $T / 4$ (solid green); (c) same as panel (b) but for helium atom and the separate contributions, short-range (circles), Coulomb-phase (squares), logarithmic (diamonds), and the total (stars) in a three-cycle streaking pulse. For all calculations, a streaking pulse of wavelength $\lambda_{s}=800 \mathrm{~nm}$ and $I_{s}=1 \times 10^{12} \mathrm{~W} / \mathrm{cm}^{2}$ has been considered.

\subsection{Role of Attochirp}

The analytical formula has also been applied to study the effects of properties of the ionizing XUV pulse on the streaking time delay. For example, it has recently been suggested [27] that attosecond time measurements may depend on the chirp of the XUV pulse. This conjecture was related to the presumption that the asymptotic kinetic energy of the photoelectron would depend on the time of transition into the continuum during the interaction with a chirped XUV pulse. To study the effect of the attochirp on the streaking time delay, the expectation value in the chirped laser pulse was determined as [28]:

$$
\left\langle\Delta t_{s}\right\rangle\left(\omega_{0}, b\right)=\int d \omega \int d t_{i} P\left(\omega, \omega_{0}, t_{i}, b\right) \Delta t_{s}\left(\omega, t_{i}\right) \simeq \int d \omega P_{\text {avg }}\left(\omega, \omega_{0}, b\right) \Delta t_{s}(\omega),
$$

where $P\left(\omega, \omega_{0}, t_{i}, b\right)$ is the probability distribution for a transition at a certain frequency $\omega$ at a given time $t_{i} ; \omega_{0}$ is the central frequency of the pulse, and $b$ is related to the chirp in the spectral distribution of a linearly chirped Gaussian pulse, 


$$
g\left(\omega, \omega_{0}, t, b\right)=\exp \left[-\frac{\left(\omega-\omega_{0}-b t\right)^{2}}{\gamma^{2} / 4 \ln 2}\right]
$$

with $\gamma^{2}=\Gamma^{2}-\frac{\tau^{2} b^{2}}{4}$, where $\Gamma=\frac{1}{\tau}\left[64(\ln 2)^{2}+\tau^{4} b^{2}\right]^{1 / 2}$ and $\tau$ is the full-width at half maximum (FWHM) pulse duration. Since it has been shown that the streaking time delay does not vary significantly with the time of ionization, in Equation (18), the first expression has been further approximated using the time averaged probability distribution $P_{\text {avg }}$.

Using the analytical estimates, Equation (11), in Equation (18) and the time averaged probability distribution for photoionization in a Gaussian pulse [29], the time delay has been evaluated as a function of the XUV central frequency $\omega_{0}$ and chirp parameter $b$. From Equation (18), one expects that the influence of the chirp is large when the time delay and/or the photoionization cross section changes rapidly as a function of the laser frequency. It is well known that the cross section varies strongly near the frequency for a Cooper minimum. Indeed, the predictions for the time delay, presented as a difference between delays obtained in a chirped and an unchirped pulse in Figure 6, show that the attochirp has a rather small effect for (a) $\mathrm{Ne}(2 p)$ while it is significant in a certain range of frequencies for $(b) \operatorname{Ar}(3 p)$. This is in agreement with the fact that the photoionization cross section for neon atom is rather smooth as a function of laser frequency. In contrast, the Cooper minimum in $\operatorname{Ar}(3 p)$ photoemission leads to variations of up to a couple of tens of attoseconds in the streaking time delay at small XUV frequencies.

\subsection{Resolution of Dynamics}

Since the streaking method accounts for the potential that the photoelectron crosses until the streaking pulse ceases, it is also interesting to study if the streaking time delay is sensitive to dynamical changes in the potential. This would open up the perspective to not only image static potentials, which are related to the conventional Wigner-Smith time delay, but also open the perspective to use the streaking method as an ultrafast imaging method. Because our analysis, c.f. Equation (8), shows that the delay consists of instantaneous field-free time delays weighted by the instantaneous streaking field strength as the photoelectron propagates through the potential, we expect that dynamics of the order of the oscillation period of the streaking field may be resolved. For a typical near-infrared streaking field, this would correspond to a resolution down to a few femtoseconds.
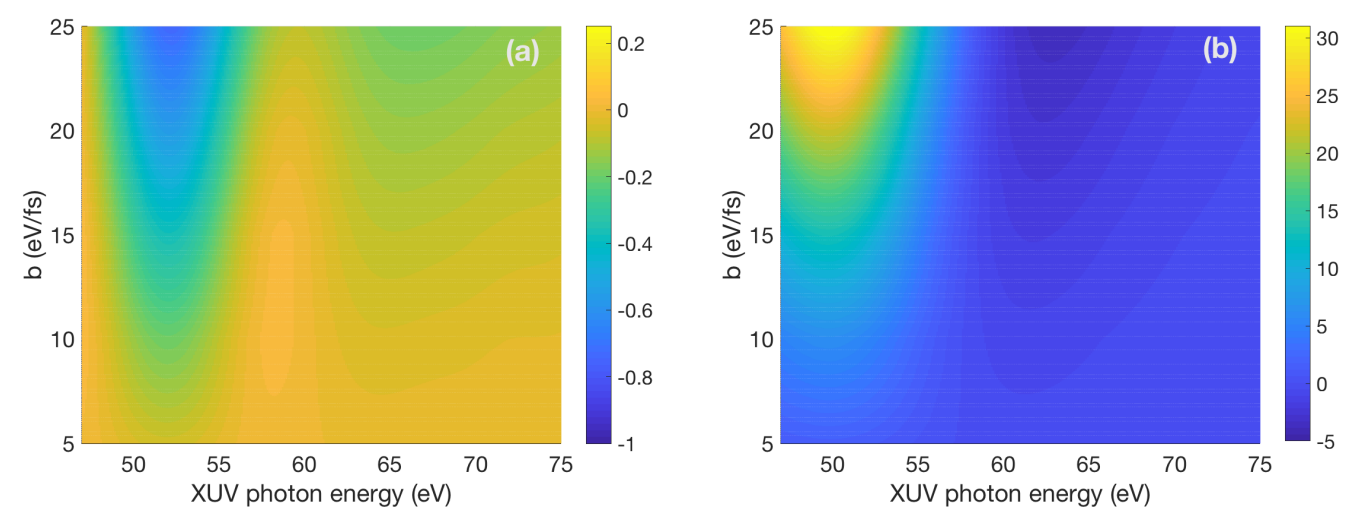

Figure 6. Difference between streaking time delays $\Delta t_{s}(b)-\Delta t_{s}(b=0)$ (in attoseconds) in a chirped to those in an unchirped pulse for photoemission from (a) $2 p$ shell of neon atom and (b) $3 p$ shell of argon atom as function of electron energy and chirp parameter.

In order to provide first insights, we have performed a model study using the 1D potential given by Equation (10), where the additional Gaussian potential now oscillated 20 a.u. in each direction about the center at $x_{0}=850$ a.u. The oscillation period of the potential was varied and for each period we performed a series of ab initio calculations, as described in Section 2.1, in order to obtain 
the streaking time delay. The results of these simulations are shown in Figure $7 \mathrm{a}$ as a function of the oscillation period of the Gaussian potential. For comparison, the time delay obtained for a static Gaussian potential is also shown. As can be seen, the time delay differs from the static value for a large oscillation period; however, at small periods below $1 \mathrm{fs}$ (for zoom-in, see Figure $7 \mathrm{~b}$ ), the time delay agrees with the static value. This indicates that, while the time delay cannot account for dynamics faster than about one-third of the period of the applied streaking field (here, $800 \mathrm{~nm}$ corresponding to a period of about $2.7 \mathrm{fs}$ ), the delay is clearly sensitive to dynamics in the few femtosecond regime.
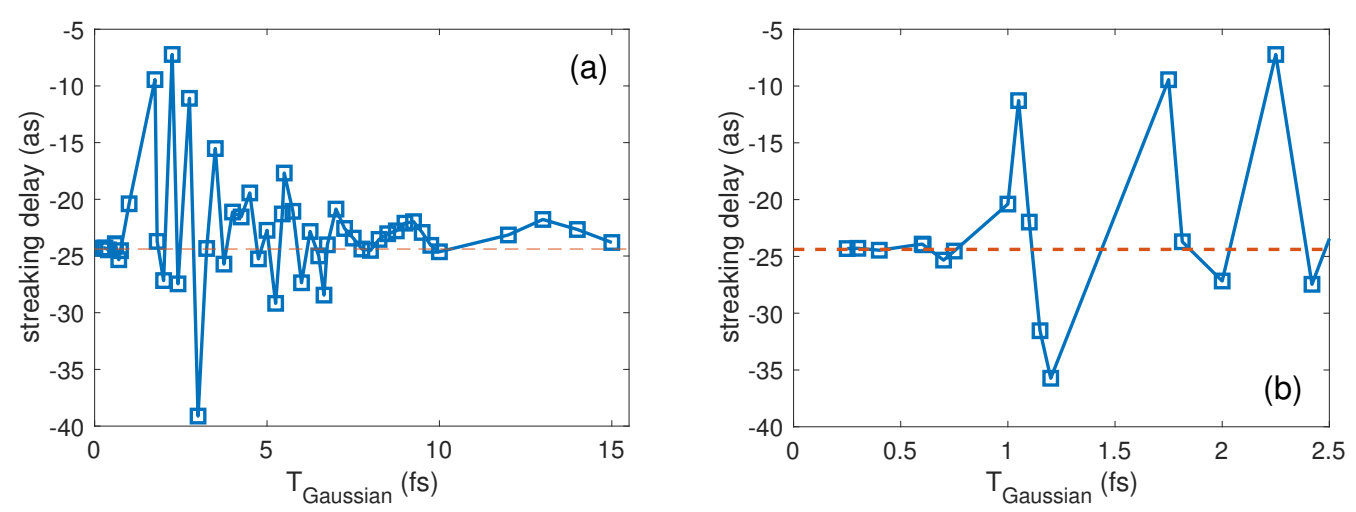

Figure 7. (a) Streaking delay as a function of the period of oscillation of the additional Gaussian potential (diamonds). The same potential and XUV pulse as in Figure 3 with a 11-cycle streaking pulse of $800 \mathrm{~nm}$ wavelength is used. Also shown is the time delay for the same parameters, but with the additional Gaussian potential remaining fixed (dotted horizontal line); (b) zoom-in of (a) for small oscillation periods.

\section{Conclusions}

We have presented a review of theoretical studies leading to the finite-range interpretation of the attosecond streaking time delay. Classical analysis of the dynamics of the photoelectron in the streaking field following absorption of the XUV photon, along with a comparison with results of ab initio quantum calculations, led to the conclusion that the streaking time delay is accumulated until the streaking field ends over a finite range of the potential of the residual ion only. This analysis further gave rise to an analytical model formula that was used to provide insights into various aspects of the streaking time delay. For example, an alternative justification into the widely used decomposition of the streaking time delay into a Wigner-Smith time delay and a Coulomb-laser coupling term has been given. The role of the attochirp of the ionizing XUV pulse as well as the relevance of the first-half cycle of the streaking field for the observed streaking time delay have been studied and discussed. Finally, we have discussed the perspective of using the streaking time delay to resolve dynamical aspects, namely temporal changes of the potential explored by the photoelectron.

Author Contributions: Conceptualization, Investigation, Methodology, Validation, Formal Analysis, C.G., A.J.-B. and A.B.; Software, Visualization, C.G.; Writing, C.G., A.J.-B. and A.B.; Supervision, Project Administration, and Funding Acquisition, A.J.-B., A.B.

Funding: This work was primarily supported (C.G., A.B) by a grant from the U.S. Department of Energy, Division of Chemical Sciences, Atomic, Molecular and Optical Sciences Program (Award No. DE-SC0001771). A.J.-B. acknowledges support by a grant from the U.S. National Science Foundation (Grant No. PHY-1734006).

Acknowledgments: We acknowledge Jing Su and Hongcheng Ni for their work on the finite-range interpretation of the streaking time delay and time delay in two-photon ionization, which has been published in Refs. [11,20,21].

Conflicts of Interest: The authors declare no conflict of interest. 


\section{References}

1. Gallmann, L.; Cirelli, C.; Keller, U. Attosecond Science: Recent Highlights and Future Trends. Annu. Rev. Phys. Chem. 2012, 63, 447-469. [CrossRef] [PubMed]

2. Calegari, F.; Sansone, G.; Stagira, S.; Vozzi, C.; Nisoli, M. Advances in attosecond science. J. Phys. B At. Mol. Opt. Phys. 2016, 49, 062001. [CrossRef]

3. Jaron-Becker, A.; Becker, A. Attosecond Spectroscopy. In Encyclopedia of Modern Optics , 2nd ed.; Guenther, R., Steel, D., Eds.; Elsevier: Amsterdam, The Netherlands, 2018; pp. 233-243, ISBN 9780128092835.

4. Itatani, J., Quéré, F.; Yudin, G.L.; Ivanov, M.Y.; Krausz, F.; Corkum, P.B. Attosecond Streak Camera. Phys. Rev. Lett. 2002, 88, 173903. [CrossRef] [PubMed]

5. Schultze, M.; Fieß, M.; Karpowitz, N.; Gagnon, J.; Korbman, M.; Hofstetter, M.; Neppl, S.; Cavalieri, A.L.; Komninos, Y.; Mercouris, T.; et al. Delay in Photoemission. Science 2010, 328, 1658-1662. [CrossRef] [PubMed]

6. Wigner, E.P. Lower Limit for the Energy Derivative of the Scattering Phase Shift. Phys. Rev. 1955, 98, 145. [CrossRef]

7. Smith, F.T. Lifetime Matrix in Collision Theory. Phys. Rev. 1960, 118, 349. [CrossRef]

8. Dahlströhm, J.M.; L'Huillier, A.; Maquet, A. Introduction to attosecond delays in photoionization. J. Phys. B At. Mol. Opt. Phys. 2012, 45, 183001. [CrossRef]

9. Maquet, A.; Caillat, J.; Taieb, R. Attosecond delays in photoionization: Time and quantum mechanics. J. Phys. B At. Mol. Opt. Phys. 2014, 47, 204004. [CrossRef]

10. Pazourek, R.; Nagele, S.; Burdörfer, J. Attosecond chronoscopy of photoemission. Rev. Mod. Phys. 2015, 87, 765. [CrossRef]

11. Su, J.; Ni, H.; Jaron-Becker, A.; Becker, A. Time Delays in Two-Photon Ionization. Phys. Rev. Lett. 2014, 113, 263002. [CrossRef]

12. Goldsmith C.; Su, J.; Jaron-Becker, A.; Becker, A. Analysis of absorption time delays of resonant and nonresonant two-photon ionization. J. Phys. B At. Mol. Opt. Phys. 2018, 51, 155602. [CrossRef]

13. Jimenez, A.; Argenti, L.; Martin, F. Modulation of Attosecond Beating in Resonant Two-Photon Ionization. Phys. Rev. Lett. 2014, 113, 263001. [CrossRef] [PubMed]

14. Pazourek, R.; Nagele, S.; Burgdörfer, J. Probing time-ordering in two-photon double ionization of helium on the attosecond time scale. J. Phys. B At. Mol. Opt. Phys. 2015, 48, 061002. [CrossRef]

15. Argenti, L.; Jiménez-Galan, A.; Caillat, J.; Taeib, R.; Martin, F. Control of photoemission delay in resonant two-photon transitions. Phys. Rev. A 2017, 95, 043426. [CrossRef]

16. Cirelli, C.; Marange, C.; Heuser, S.; Petersson, C.L.M.; Jimenez-Galan, A.; Argenti, L.; Zhong, S.; Busto, D.; Isinger, M.; Nandi, S.; et al. Anisotropic photoemission time delays close to a Fano resonance. Nat. Commun. 2018, 9, 955. [CrossRef]

17. Isinger, M.; Squibb, R.J.; Busto, D.; Zhong, S.; Harth, A.; Kroon, D.; Nandi, S.; Arnold, C.L.; Miranda, M.; Dahlström, J.M.; et al. Photoionization in the time and frequency domain. Science. 2017, 358, 893-896. [CrossRef]

18. Paul, P.M.; Toma, E.S.; Breger, P.; Mullot, G.; Auge, F.; Balcou, P.; Muller, H.G.; Agostini, P. Observation of a Train of Attosecond Pulses from High Harmonic Generation. Science 2001, 292, 1689-1692 [CrossRef]

19. Nagele, S.; Pazourek, R.; Feist, J.; Doblhoff-Dier, K.; Lemell, C.; Tökeski, K.; Burgdörfer, J. Time-resolved photoemission by attosecond streaking: Extraction of time information. J. Phys. B At. Mol. Opt. Opt. Phys. 2011, 44, 081001. [CrossRef]

20. Su, J.; Ni, H.; Becker, A.; Jaroń-Becker, A. Finite-range time delays in numerical attosecond-streaking experiments. Phys. Rev. A 2013, 88, 023413. [CrossRef]

21. Su, J.; Ni, H.; Becker, A.; Jaron-Becker, A. Attosecond-streaking time delays: Finite-range property and comparison of classical and quantum approaches. Phys. Rev. A 2014, 89, 013404. [CrossRef]

22. Su, J. Theoretical Analysis and Numerical Simulation of Attosecond Time Delays in Photoionization. Ph.D. Thesis, University of Colorado, Boulder, CO, USA, 2014.

23. Goldsmith, C.; Su, J.; Becker, A.; Jaroń-Becker, A. Analytical estimates of attosecond streaking time delay in photoionization of atoms. Phys. Rev. A 2017, 96, 053410. [CrossRef]

24. Pazourek, R.; Nagele, S.; Burgdörfer, J. Time-resolved photoemission on the attosecond scale: opportunities and challenges. Faraday Discuss. 2013, 163, 353-376. [CrossRef] [PubMed]

25. Feist, J.; Zatsarinny, O.; Nagele, S.; Pazourek, R.; Burgdörfer, J.; Guan, X.; Bartschat, K.; Schneider, B.I. Time delays for attosecond streaking in photoionization of neon. Phys. Rev. A 2014, 89, 033417. [CrossRef] 
26. Kiesewetter, D.; Jones, R.R.; Camper, A.; Schoun, S.B.; Agostini, P.; DiMauro, L.F. Probing electronic binding potentials with attosecond photoelectron wavepackets. Nat. Phys. 2017, 14, 68. [CrossRef]

27. Sabbar, M.; Heuser, S.; Boge, R.; Lucchini, M.; Carette, T.; Lindroth, E.; Gallmann, L.; Cirelly, C.; Keller, U. Resonance Effects in Photoemission Time Delays. Phys. Rev. Lett. 2015, 115, 133001. [CrossRef] [PubMed]

28. Goldsmith, C.; Su, J.; Jaroń-Becker, A.; Becker, A. Effect of attochirp on attosecond streaking time delay in photoionization of atoms. J. Phys. B At. Mol. Opt. Phys. 2018, 51, 025601. [CrossRef]

29. Krasovskii, E.E.; Bonitz, M. Towards a theory of an attosecond transient recorder Phys. Rev. A 2009, 80, 053421. [CrossRef]

(C) 2019 by the authors. Licensee MDPI, Basel, Switzerland. This article is an open access article distributed under the terms and conditions of the Creative Commons Attribution (CC BY) license (http://creativecommons.org/licenses/by/4.0/). 